\title{
Aspects of the Coarse-Grained-Based Approach to a Low-Relativistic Fractional Schrödinger Equation
}

\author{
J. Weberszpil ${ }^{a}$, C.F.L. Godinho ${ }^{b}$, A. Cherman ${ }^{c, d}$ and J.A. Helayël-Neto ${ }^{d}$ \\ ${ }^{a}$ Instituto Multidisciplinar, Departamento de Tecnologia e Linguagens \\ Universidade Federal Rural do Rio de Janeiro-UFRRJ \\ Av. Governador Roberto Silveira s/n- Nova Iguaçú, Rio de Janeiro, Brasil \\ ${ }^{b}$ Grupo de Física Teórica, Departamento de Física \\ Universidade Federal Rural do Rio de Janeiro-UFRRJ \\ BR 465-07, 23890-971, Seropédica, Rio deJaneiro, Brasil \\ ${ }^{c}$ Fundação Planetário da Cidade do Rio de Janeiro \\ Rua Vice-Governador Rubens Bernardo, 100, 22451-070, Rio de Janeiro, Brasil \\ ${ }^{d}$ Centro Brasileiro de Pesquisas Físicas-CBPF \\ Rua Dr Xavier Sigaud 150, 22290-180, Rio de Janeiro, Brasil \\ E-mail: josewebe@ufrrj.br, crgodinho@ufrrj.br, \\ achermanepcrj.rj.gov.br, helayelecbpf.br
}

\begin{abstract}
The main goal of this paper is to set up the coarse-grained formulation of a fractional Schrödinger equation that incorporates a higher (spatial) derivative term which accounts for relativistic effects at a lowest order in momentum. The corresponding continuity equation is worked out and we also identify the contribution of the relativistic correction to the quantum potential in the coarse-grained treatment. As a consequence, in the classical regime, we derive the sort of fractional Newtonian law with the quantum potential included. The fractional counterparts of the De Broglie's energy and momentum relations are also presented and discussed. An attempt to formulate equations that describe dispersion relations for massless particles and refraction index for a coarse-grained medium is presented and suggestions to include ultra-high-energetic cosmic ray (UHECR) sources, distant astrophysical sources such as Gamma-Ray Bursts (GRBs) and active galactic nuclei (AGNs) are given as possible frameworks for tests.
\end{abstract}

7th Conference Mathematical Methods in Physics - Londrina 2012,

16 to 20 April 2012

Rio de Janeiro, Brazil 


\section{Introduction}

Physicists are presently seeking and trying to understand the connections between complex systems, nonlocal field theories and other areas of Physics. Today, this is an important subject of studies in different physical and mathematical areas, but the understanding of non-linear processes connected to these topics has had a considerable boost over the past 40 years. This deeper comprehension has been inspired by the discovery and the insight of a new phenomenon, known as dynamical chaos. The main motivation is that the use of these theories may yield a much more elegant and effective treatment of problems in particle and high energy physics, as it has hitherto been carried out with the help of the local field theories. A particular subclass of nonlocal field theories is described with the operators of a fractional nature and is specified in the framework of fractional calculus (FC). The latter provides us with a set of mathematical tools to generalize the concept of derivative and integral operators with integer order to their respective extensions of an arbitrary real order. FC has raised up a great deal of interest over recent years and has been used as an applied tool to study of fractional dynamics in many fields of physics, mechanics, engineering and other areas to approach problems connected with complex systems [1]. Today, there is a rich stream of works linking such areas throughout different paths, [2]. Nonlocal theories and memory effects can also be connected to complexity and admit a treatment in terms of FC. In this context, the nondifferentiable nature of the microscopic dynamics may be connected with time scales so as to approach questions in the realm of complex systems [3].

Various important characteristics of a quantum system may not be well determined if the relativistic effects are not completely taken into account in the calculations, as it can be seen in the context of several areas of research in Physics. Including relativistic effects may lead to significant differences in the results. Without taking into account these effects, serious inaccuracies may shows up. For example, it is important in the context of the atomic and molecular structure to describe energy levels of excited states and the fine structure of hydrogen-like atoms. Clarifying, in the calculation of hydrogen atom, sufficient experimental accuracy requires that relativistic effects must be taken into account [4]. These relativistic effects can be quite significant to the correct computation of the atomic spectrum and in the study of heavy-ion collisions, where relativistic contributions are typically much larger especially for atoms with large nuclear charges $Z e$. The inclusion of relativistic effects into the Schrödinger equation has been considered in the studies of the electron motion in the operation of free-electron lasers [5]. This approach is also important for the interpretation of highly accurate experiments in spectroscopy. For heavy atoms and their compounds, relativistic effects do an important role. This is related to the fact that these effects on the energies and other physical quantities increase with the fourth power of the nuclear charge Ze [6]. On the other hand, there are cases in which estimates of a good accuracy may not need the inclusion of relativistic corrections. Some criteria for judging the importance of including relativistic effects should be determined to to compute the influence of relativity on the structure and on the strength of the effects of the systems under study. This is because a fully relativistic model may lead to 
more extensive calculations than the non-relativistic counterpart, as for instance atomic structures indicate [4].

The Klein-Gordon (KG) and the Dirac equations were proposed as relativistic versions of the linear Schrödinger equation. After a few decades of their introduction in Particle Physics, they have been reconsidered to study relativistic quark-anti-quark bound states [7] and the gravitational collapse of boson stars [8].

In the present contribution, we pursue an investigation of the coarse-grained fractional Schrödinger equation, corrected by a fourth spatial derivative term which accounts for lowest power in momentum (relativistic) correction to the kinetic energy term. To do that, we should mention some considerations of a more formal nature.

Recently, an alternative definition to the Riemann-Liouville fractional derivative, called the modified Riemann-Liouville fractional derivative (MRL) [9], has been proposed with the advantages of both the standard Riemann-Liouville and Caputo fractional derivatives: it is defined for arbitrary continuous (nondifferentiable) functions and the fractional derivative of a constant being zero. The MRL approach seems to give a mathematical framework for dealing with dynamical systems defined on coarse-grained spaces and/or with coarse-grained time, since the use of fractional calculus appears to be intimately related to fractal and self-similar functions. The well-tested definitions for fractional derivatives, namely, Riemann-Liouville and Caputo have been frequently used for several applications. In spite of their adequacy, they present some dangerous pitfalls. Basic definitions $[3,9,10,11,12,13]$ and details on the formalism may be found in the quoted papers and references therein.

We would like to emphasize that the choice of the MRL approach, besides the points already mentioned, is justified by the fact that the chain and Leibniz rules acquires a simpler form, which helps a great deal if changes of coordinates are performed. Moreover, causality seems to be more easily obeyed in a field-theoretical construction if we adopt this approach. For theses reasons, we adopt here the MRL approach for fractional derivatives.

Here, we claim that the use of an approach based on a sequential form of MRL [9] is more appropriate to describe the dynamics associated with field theory and particle physics in the space of nondifferentiable solution functions, or in the coarse-grained space-time. Based on this approach, we have worked out a suggested version of a fractional Schrödinger equation, with a lowest-order relativistic correction, obtained from a fractional wave equation [3] to which a mass term has been adjoined, to give us a fractional Klein-Gordon equation (FKGE). With the definition of some fractional operators, the McLaurin expansion and an ansatz for the plane wave solutions, we have obtained fractional versions of Bohmian equations to describe the particle dynamics associated with Bohmian mechanics, in the space of non-integer differentiable functions. We had also done a formulation for an anomalous dispersion relation and to a refraction index, related to massless particle in a coarse-grained media and a vacuum refractive index for a coarse-grained non-trivial optical medium.

In a previous work [3], we have argued that the modeling of Te V-physics may demand an 
approach based on fractal operators and FC and we claimed that, in the realm of complexity, nonlocal theories and memory effects were connected to complexity and also that the FC and the nondifferentiable nature of the microscopic dynamics may be connected with time scales. Using the MRL definition of fractional derivatives, we have worked out explicit solutions to a fractional wave equation with suitable initial conditions to carefully understand the time evolution of classical fields with fractional dynamics. First, by considering space-time partial fractional derivatives of the same order in time and space, a generalized fractional D'Alembertian is introduced. By means of a transformation of variables to light-cone coordinates, an explicit analytical solution was obtained. Also, aspects connected with Lorentz symmetry were analyzed with two different approaches.

It seems that a reasonable way to probe the classical framework of physics is to remark that, in the space of our real world, the generic point is not infinitely small (or thin), it rather has a thickness. In a coarse-grained space, a point is not infinitely thin, and here, this feature is modeled by means of a space in which the generic differential is not $d x$, but rather $(d x)^{\alpha}$, and likewise for the time variable t. It is noteworthy to highlight the ideas in the interesting work by Nottale [14], where the notion of fractal space-time is first introduced. Non-integer differentiability and randomness are mutually related in their nature, in such a way that studies on fractals on the one hand, and fractional Brownian motion on the other hand, are often parallel in the ref. [14]. A function continuous everywhere, but nowhere integer-differentiable, necessarily exhibits randomlike or pseudo-random features, in that various samplings of these functions, on the same given interval, will be different. This may explain the huge amount of literature extending the theory of stochastic differential equations to describe stochastic dynamics driven by fractional Brownian motion $[9,10,13]$.

Our paper is outlined as follows: In Section 2, we review the development of the low-relativistic correction to the integer order Schrödinger equation and discuss the FKGE. In Section 3, we work out the low-relativistic fractional Schrödinger equation. The fractional continuity equation is the subject of the section 4. Section 5 is devoted to the development of the fractional Bohmian equations with low-relativist limit. Section 6 is devoted to applications. Finally, in Section 7, we cast our Concluding Comments and prospects for further investigation.

\section{Lowest-Order Relativistic Corrections to the Integer Schrödinger Equation and the Fractional Klein Gordon Equation}

We start off from the well-known relativistic relation,

$$
E=\sqrt{\vec{p}^{2} c^{2}+m^{2} c^{4}},
$$


where,

$$
\left\{\begin{array}{ll}
E= & m_{0} c^{2} \gamma ; \\
\vec{p}= & m_{0} \vec{v} \gamma
\end{array} \quad \gamma=\frac{1}{\sqrt{1-\frac{v^{2}}{c^{2}}}}\right.
$$

We readily get that $\frac{\vec{p} c}{E}=\frac{\vec{v}}{c}$. So, in the non-relativistic regime $(|\vec{v}| \ll c),|\vec{p}| c \ll E$, and so the following approximation can be adopted:

$$
\begin{aligned}
E-m c^{2} & \equiv \varepsilon_{n r} \cong \frac{p^{2}}{2 m}-\frac{p^{4}}{8 m^{3} c^{2}}= \\
& =\frac{(p c)^{2}}{2 m c^{2}}\left[1-\left(\frac{p c}{2 m c^{2}}\right)^{2}\right],
\end{aligned}
$$

where $\varepsilon_{n r}$ stands for the non-relativistic kinetic energy. Here, it is worthy noting that the lowest-order relativistic limit corresponds to momenta such that $|\vec{p}| c \ll 2 m c^{2}$, that is the threshold energy for a pair creation. The fractional approach may be justified if we argue that the particle described by this formalism is actually a pseudo-particle that carries information of the medium and particular interaction implicit in the equation that describes its evolution. This pseudo-particle is then "dressed" with information about medium and interactions, and the solutions to the fractional equation, like the Green functions in condensed matter physics, carry additional information about interactions and media. Then, even if the medium is not fractal, the fractional approach still makes sense to describe the evolutions of a pseudo-particle.

Now, by adopting the correspondence principle

$$
\left\{\begin{array}{l}
\widehat{E}=i \hbar \frac{\partial}{\partial t} \\
\widehat{p}=-i \hbar \frac{\partial}{\partial x}
\end{array} ;\right.
$$

we obtain the Schrödinger with a lowest-order relativistic correction in momentum; it reads

$$
i \hbar \frac{\partial}{\partial t} \psi(x, t)=-\left(\frac{\hbar^{2}}{2 m}\right) \frac{\partial^{2}}{\partial x^{2}} \psi(x, t)-\left(\frac{\hbar^{4}}{8 m^{3} c^{2}}\right) \frac{\partial^{4}}{\partial x^{4}} \psi(x, t)+V \psi(x, t) .
$$

With this extra term in Schrödinger equation, we then construct, in the sequel, the FKGE with a mass term. In the following subsection we shall be doing that with the aim of extracting the non-relativistic regime of the $\mathrm{KG}$ equation, from which the fourth power correction in momentum naturally follow.

\section{The Fractional Klein Gordon Equation}

In a recent paper [3], we have obtained in a natural way the fractional wave equation. Now, we shall write down a fractional version of the KG equation by the addition of the mass term to 
the fractional wave equation, considering adequate dimension scale factors, in order to gain some insight to about the fractional quantum operator to be used.

The usual KG equation reads

$$
\frac{1}{c^{2}} \frac{\partial^{2}}{\partial t^{2}} \psi(x, t)-\frac{\partial^{2}}{\partial x^{2}} \psi(x, t)+\frac{m^{2} c^{2}}{\hbar^{2}} \psi(x, t)=0 .
$$

Fractional Klein-Gordon equation [15] and fractional Dirac equation have been studied by several authors over the past decade $[16,17,18]$. Some articles have been dealing with fractional power of D'Alembertian operator used in the non local kinetic terms Lagrangian field theory in the (2+1)-dimensional bosonization and also to study the effective field theory, which has some degrees of freedom integrated out from the underlying local theory [19, 20, 21]. The canonical quantization of fractional massless and massive fields has been studied by some authors [2, 22, 23] and quantization of fractional KG field and fractional gauge field based on Nelson's stochastic mechanics and Parisi-Wu stochastic quantization procedure at zero and positive temperature have been considered $[24,25]$. An axiomatic approach to fractional KG field, where properties of the $n$ point Schwinger or Euclidean Green functions and their analytic continuation to the corresponding n-point Wightman functions were studied by [26, 27].

The fractional KG equation can be written here, in an similar manner as in ref. [28], but with different fractional orders in space and time, as below:

$$
\frac{1}{c^{2 \beta}} \frac{\partial^{2 \beta}}{\partial t^{2 \beta}} \psi(x, t)-M_{x, \alpha}^{2} \frac{\partial^{2 \alpha}}{\partial x^{2 \alpha}} \psi(x, t)+\frac{m^{2 \beta} c^{2 \beta}}{\hbar^{2 \beta}} \psi(x, t)=0 .
$$

The diffusion factor, $M_{x, \alpha}$, is here introduced for dimensional consistency reasons. This equation has also to be consistent with an fractional relativistic energy-momentum equation, given by

$$
E^{\beta}=\sqrt{p^{2 \alpha} c^{2 \alpha}+m^{2 \beta} c^{4 \beta}} .
$$

Now, with these considerations, we shall expand the momentum energy of eq.(2.8)in terms of an integer McLaurin's series and, after the substitution of fractional quantum operators, obtain the fractional Schrödinger equation with lowest-order relativistic correction term.

\section{Fractional Schrödinger Equation with Lowest-Order Relativistic Correction}

A method first used for the attainment of a fractional Schrödinger equation was formalism of the path integral over the Lèvy paths [29, 30], where a fractional generalization of the Schrödinger equation in terms of the quantum Riesz fractional derivative was obtained to study the energy spectra of hydrogen-like atoms, a fractional oscillator in the semi-classical approximation and the validity of the parity conservation law. The argument to write down the fractional Schrödinger equation [31] was that the path integral over Brownian trajectories leads to the ordinary Schrödinger equation, while the path integral over Lèvy trajectories should lead to the space fractional Schrödinger 
equation. Other versions of Schrödinger equation were obtained [32] considering only a time fractional Schrödinger equation in the sense of a Caputo fractional time derivative formalism. A version of the generalized fractional Schrödinger, with space-time fractional derivatives in the sense of Caputo and Riesz fractional derivatives, was studied in ref. [33] and solved for free particle and square well potential with integral transform methods. The fractional Schrödinger equation can also be obtained by methods like a fractional variational method in the context of a Lagrangian formulation or by a FKGE[28].

Here, we adopt the MRL approach for fractional derivatives, which is less restrictive than other definitions, to obtain the lowest-order relativistic correction to a fractional Schrödinger equation, with different orders for the fractional derivatives in time and space. This is carried out with the use of a FKGE.

The main rules used here, with the MRL approach, are summarized as:

$D^{\alpha} K=0, K$ is constant, $D^{\alpha} x^{\gamma}=\frac{\Gamma(\gamma+1)}{\Gamma(\gamma+1-\alpha)} x^{\gamma-\alpha}, \gamma>0$, derivative of power function, $(u(x) v(x))^{(\alpha)}=$ $u^{(\alpha)}(x) v(x)+u(x) v^{(\alpha)}(x)$ is the Leibniz rule. The chain rule for non differentiable functions is written as

$$
\frac{d^{\alpha}}{d x^{\alpha}} f[u(x)]=\frac{d^{\alpha} f}{d u^{\alpha}}\left(\frac{d}{d x} u\right)^{\alpha},
$$

where $f$ is $\alpha$-differentiable and $u$ is differentiable with respect to $x$ and, for coarse-grained spacetime as

$$
\frac{d^{\alpha}}{d x^{\alpha}} f[u(x)]=\frac{d f}{d u} \frac{d^{\alpha}}{d x^{\alpha}} u
$$

where $f(u(x))$ is not differentiable w.r.t $x$ but it is differentiable w.r.t $u$, and $u$ is not differentiable w.r.t $x$.

For further details, the readers can follow the refs. [11, 12] which contain all the basic for the formulation of a fractional differential geometry in coarse-grained space, and refers to an extensive use of coarse-grained phenomenon.

Its is worthy to point out that the Leibniz rule used here is a good approximation that comes from the first two terms of the fractional Taylor series development, that holds only for nondifferentiable functions [12] and are as good and approximated as the classical integer one. Here, a comment is pertinent: the fractional MRL approach for nondifferentiable functions has similar rules and has definition with a mathematical limit operation comparable to certain definitions of local fractional derivatives, as that introduced by Kolwankar and Gandal [34, 35, 36] with some studies in the literature. For example, the works of Refs. [37, 38, 39] or the approaches with Hausdorff derivative, also called fractal derivative [40, 41], that can be applied to power-law phenomena and the recently developed $\alpha$-derivative [42]. The MRL approach seems to us to be an integral version of the calculus mentioned above and all of them deserve to be more deeply investigated, under a mathematical point of view, in order to give exact differences and similarities respect to the traditional fractional calculus with Riemann-Liouville or Caputo definition and with local frac- 
tional calculus and even fractional q-calculus [1, 43, 44, 47], as well as in the comparative point of view of physics[41, 44, 47, 48, 49], for the scope of applicability.

We think that the referred alternative formalisms can be used to the attainment of results similar or with similarities to some of those here obtained [50] and this is a good indication that our results are more general and not only dependent and provided by an specific formalism. With these considerations we proceed now to construct the fractional Schrödinger equation with lowest-order relativistic correction.

We develop eq.(2.8) in McLaurin's series by taking $f(x)=\left(1+x_{\alpha, \beta}\right)^{1 / 2}$ and assuming that $f^{(\alpha k)}(x)$ has a sequential character like

$$
\begin{gathered}
f^{(2 \alpha)}(x)=\frac{\partial^{2 \alpha}}{\partial x^{2 \alpha}}=\frac{\partial^{\alpha}}{\partial x^{\alpha}} \frac{\partial^{\alpha}}{\partial x^{\alpha}} \\
f(x)=\left(1+x_{\alpha, \beta}\right)^{1 / 2} \cong 1+\frac{1}{2} x_{\alpha, \beta}-\frac{1}{8} x_{\alpha, \beta}^{2}+\mathscr{O}\left(x_{\alpha, \beta}^{3}\right) .
\end{gathered}
$$

Since the semi group properties for fractional derivatives do not hold in general, we use the Miller-Ross sequential derivative [51] in the MRL sense. Incidentally, the Miller-Ross sequential derivative is a systematic procedure that carries out a fractional higher-order derivative while avoiding the recursive application of many single derivatives taken after each other. Moreover, we took the option to carry out the sequence of derivatives in the cascade form, in MRL sense, as done in the work of ref. [12, 13].

Here, we propose the operators:

$$
\left\{\begin{array}{l}
\widehat{E}^{\beta}=i(\hbar)^{\beta} \frac{\partial^{\beta}}{\partial t^{\beta}} \\
\widehat{p}^{\alpha}=-i(\hbar)^{\alpha} M_{x, \alpha} \frac{\partial^{\alpha}}{\partial x^{\alpha}}
\end{array} ;\right.
$$

it can be verified that the fractional quantum operator proposed above, when substituted into the equation eq.(2.8), will give the KG equation eq.(2.7). Note that $M_{x, \alpha}$ factor becomes dimensionless when $\alpha$ equal to 1 and its mass dimension is in general $\alpha$-dependent.

Now, factoring out the term $m^{\beta} c^{2 \beta}$ in the eq.(2.8) and expanding in therms of McLaurin's series in $x_{\alpha, \beta}=\frac{p^{2 \alpha} c^{2 \alpha}}{m^{2 \beta} c^{4 \beta}}$, by substitution of the fractional operators in eq. (3.5), we are lead to one possible representation of the fractional Schrödinger equation given by

$$
\begin{aligned}
i(\hbar)^{\beta} \frac{\partial^{\beta} \psi(x, t)}{\partial t^{\beta}} & =-M_{x, \alpha}^{2} \frac{\hbar^{2 \alpha}}{2 m^{\beta}} \frac{c^{2 \alpha}}{c^{2 \beta}} \frac{\partial^{2 \alpha} \psi(x, t)}{\partial x^{2 \alpha}}+V_{\alpha, \beta} \psi(x, t)+ \\
& -\frac{1}{8} M_{x, \alpha}^{4} \frac{\hbar^{4 \alpha}}{m^{3 \beta}} \frac{c^{4 \alpha}}{c^{6 \beta}} \frac{\partial^{4 \alpha} \psi(x, t)}{\partial x^{4 \alpha}}
\end{aligned}
$$

where the notation is assumed $\frac{\partial^{4 \alpha}}{\partial x^{4 \alpha}}=\frac{\partial^{\alpha}}{\partial x^{\alpha}} \frac{\partial^{\alpha}}{\partial x^{\alpha}} \frac{\partial^{\alpha}}{\partial x^{\alpha}} \frac{\partial^{\alpha}}{\partial x^{\alpha}}, \frac{\partial^{\alpha}}{\partial x^{2 \alpha}}=\frac{\partial^{\alpha}}{\partial x^{\alpha}} \frac{\partial^{\alpha}}{\partial x^{\alpha}}$, since the semi-group properties for additive in the orders of the derivatives may not hold, as previously commented. 


\section{Fractional Continuity Equation}

It is well-known that, in ordinary quantum mechanics, the continuity equation has a very important role, it describes probability conservation law. But, in the context of a fractional quantum mechanics, the meaning of a fractional continuity equation is not quite clear and requires some analysis. Since we are in the interaction picture and dealing with pseudo-particles or "dressed" particles, the fractional continuity equation could reveal that there exists an implicit dissipation implicit in the fractional evolution equations. Specially, if the orders of derivatives in space and time are different from each other. This could mean that the fractional equations can be thought as related to some effective theories. The known and unknown information about interactions and the media could be accounted for in the fractionality. Whenever the integer order limit for derivatives is reached, the conservation law emerges, dissipation is no more present in the theory and certain symmetries could be restored. We expect that future scientific investigations may clarify more the real meaning of the fractional continuity equation.

To obtain our fractional continuity equation, we now proceed as follows: the conjugate of fractional Schrödinger equation is taken:

$$
\begin{aligned}
-i(\hbar)^{\beta} \frac{\partial^{\beta} \psi(x, t)}{\partial t^{\beta}} & =-M_{x, \alpha}^{2} \frac{\hbar^{2 \alpha}}{2 m^{\beta}} \frac{c^{2 \alpha}}{c^{2 \beta}} \frac{\partial^{2 \alpha} \psi(x, t)}{\partial x^{2 \alpha}}+V_{\alpha, \beta} \psi(x, t)+ \\
& -\frac{1}{8} M_{x, \alpha}^{4} \frac{\hbar^{4 \alpha}}{m^{3 \beta}} \frac{c^{4 \alpha}}{c^{6 \beta}} \frac{\partial^{4 \alpha} \psi(x, t)}{\partial x^{4 \alpha}}
\end{aligned}
$$

Probability is defined as usually: $P=\psi^{*}(x, t) \psi(x, t)$.

Multiplying (3.6) by $\psi^{*}(x, t)$ and equation (4.1) by $-\psi(x, t)$, after adding both equations, we obtain

$$
\begin{array}{r}
i(\hbar)^{\beta} \frac{\partial^{\beta}}{\partial t^{\beta}}\left(\psi^{*}(x, t) \psi(x, t)\right)= \\
-M_{x, \alpha}^{2} \frac{\hbar^{2 \alpha}}{2 m^{\beta}} \frac{c^{2 \alpha}}{c^{2 \beta}}\left[\psi^{*}(x, t) \frac{\partial^{2 \alpha} \psi(x, t)}{\partial x^{2 \alpha}}-\psi(x, t) \frac{\partial^{2 \alpha} \psi^{*}(x, t)}{\partial x^{2 \alpha}}\right]+ \\
-\frac{1}{8} M_{x, \alpha}^{4} \frac{\hbar^{4 \alpha}}{m^{3 \beta}} \frac{c^{4 \alpha}}{c^{6 \beta}}\left[\psi^{*}(x, t) \frac{\partial^{4 \alpha} \psi(x, t)}{\partial x^{4 \alpha}}-\psi(x, t) \frac{\partial^{4 \alpha} \psi^{*}(x, t)}{\partial x^{4 \alpha}}\right] .
\end{array}
$$

After some algebra, the latter equation can be written as

$$
\frac{\partial^{\beta} \rho(x, t)}{\partial t^{\beta}}+\frac{\partial^{\alpha} J(x, t)}{\partial x^{\alpha}}=0
$$

where $\rho(x, t) \equiv \psi^{*}(x, t) \psi(x, t)$ and 


$$
\begin{aligned}
J= & M_{x, \alpha}^{2} \frac{\hbar^{2 \alpha}}{2 m^{\beta} i \hbar^{\beta}} \frac{c^{2 \alpha}}{c^{2 \beta}} J^{\prime}+ \\
& +\frac{1}{8} M_{x, \alpha}^{4} \frac{\hbar^{4 \alpha}}{m^{3 \beta} i \hbar^{\beta}} \frac{c^{4 \alpha}}{c^{6 \beta}} \frac{\partial^{\alpha}}{\partial x^{\alpha}}\left\{\left[J^{\prime}-2\left(\frac{\partial^{\alpha} \psi^{*}(x, t)}{\partial x^{\alpha}} \frac{\partial^{\alpha} \psi(x, t)}{\partial x^{\alpha}}\right)\right]+4\left(\frac{\partial^{\alpha} \psi(x, t)}{\partial x^{\alpha}} \frac{\partial^{2 \alpha} \psi^{*}(x, t)}{\partial x^{2 \alpha}}\right)\right\},
\end{aligned}
$$

with $J^{\prime} \equiv\left[\psi^{*}(x, t) \frac{\partial^{\alpha} \psi(x, t)}{\partial x^{\alpha}}-\psi(x, t) \frac{\partial^{\alpha} \psi^{*}(x, t)}{\partial x^{\alpha}}\right]$.

Equation (4.3) shows that the probability is conserved in the fractional sense.

Taking $\alpha=\beta=1$, we obtain the integer continuity equation with the lowest-order relativistic correction.

\section{Fractional Quantum Potential with Lowest-Order Relativistic Correction terms}

Now, we shall build up the fractional Bohmian equations, by parameterizing the solution of eq.(3.6) as below:

$$
\Psi(\mathbf{r}, t)=R(\mathbf{r}, t) e^{i S(\mathbf{r}, t) / \hbar}
$$

where $R$ and $S$ are the amplitude of probability density and phase of $\Psi$, respectively, both being real-valued functions. Substituting this relation into the fractionalSchrödinger's equation and multiplying by $e^{-i S(\mathbf{r}, t) / \hbar}$, after some algebra and taking real and imaginary parts, we get two equations that lead to a fractional version of Bohmian Mechanics, including the its lowest-order relativistic correction limit.

Now, proceeding as described above, two equations are obtained:

a) for the real part:

$$
\begin{aligned}
-M_{x, \alpha}^{2} \frac{\hbar^{2 \alpha}}{2 m^{\beta}} \frac{c^{2 \alpha}}{c^{2 \beta}} \frac{1}{R(x, t)} \frac{\partial^{\alpha}}{\partial x^{\alpha}} \frac{\partial^{\alpha} R}{\partial x^{\alpha}}+M_{x, \alpha}^{2} \frac{\hbar^{2 \alpha}}{2 m^{\beta}} \frac{c^{2 \alpha}}{c^{2 \beta}} \frac{1}{\hbar^{2}}\left(\frac{\partial^{\alpha} S}{\partial x^{\alpha}}\right)^{2}+\hbar^{\beta-1} \frac{\partial^{\beta} S}{\partial t^{\beta}}+V+ \\
-\frac{1}{8} M_{x, \alpha}^{4} \frac{\hbar^{4 \alpha}}{m^{3 \beta}} \frac{c^{4 \alpha}}{c^{6 \beta}} \frac{1}{R}\left[\frac{1}{\hbar^{4}} R\left(S^{(\alpha)}\right)^{4}+R^{(4 \alpha)}-\frac{4}{\hbar^{2}} R S^{(\alpha)} S^{(3 \alpha)}+\right. \\
\left.-\frac{12}{\hbar^{2}} R^{(\alpha)} S^{(\alpha)} S^{(2 \alpha)}-\frac{3}{\hbar^{2}} R^{2}\left(S^{(2 \alpha)}\right)^{2}-\frac{6}{\hbar^{2}} R^{(2 \alpha)}\left(S^{(\alpha)}\right)^{2}\right]=0
\end{aligned}
$$


b) for the imaginary part:

$$
\begin{gathered}
\frac{\partial^{\beta} R^{2}}{\partial t^{\beta}}+2 M_{x, \alpha}^{2} \frac{1}{2 m^{\beta}} \frac{c^{2 \alpha}}{c^{2 \beta}} \hbar^{2 \alpha-\beta-1} \frac{\partial^{\alpha}}{\partial x^{\alpha}}\left(R^{2} \frac{\partial^{\alpha} S}{\partial x^{\alpha}}\right)+ \\
-\frac{1}{8} M_{x, \alpha}^{4} \frac{\hbar^{4 \alpha}}{m^{3 \beta}} \frac{c^{4 \alpha}}{c^{6 \beta}} \frac{i}{\hbar^{4}}\left(\frac{-2 R}{\hbar^{\beta}}\right)\left[R \hbar^{3} S^{(4 \alpha)}-4 \hbar R^{(\alpha)}\left(S^{(\alpha)}\right)^{3}+4 \hbar^{3} R^{(\alpha)} S^{(3 \alpha)}+6 \hbar^{3} R^{(2 \alpha)} S^{(2 \alpha)}\right]=0 .
\end{gathered}
$$

The first term in the left-hand side of eq.(5.2) can be called fractional quantum potential due to the presence of Planck constant and fractional derivatives:

$$
\begin{gathered}
Q^{\alpha}(x, t) \equiv-M_{x, \alpha}^{2} \frac{\hbar^{2 \alpha}}{2 m^{\beta}} \frac{c^{2 \alpha}}{c^{2 \beta}} \frac{1}{R} \frac{\partial^{\alpha}}{\partial x^{\alpha}} \frac{\partial^{\alpha} R}{\partial x^{\alpha}}+ \\
-\frac{1}{8} M_{x, \alpha}^{4} \frac{\hbar^{4 \alpha}}{m^{3 \beta}} \frac{c^{4 \alpha}}{c^{6 \beta}} \frac{1}{R}\left[R^{(4 \alpha)}-\frac{4}{\hbar^{2}} R S^{(\alpha)} S^{(3 \alpha)}-\frac{12}{\hbar^{2}} R^{(\alpha)} S^{(\alpha)} S^{(2 \alpha)}-\frac{3}{\hbar^{2}} R^{2}\left(S^{2 \alpha)}\right)^{2}-\frac{6}{\hbar^{2}} R^{(2 \alpha)}\left(S^{(\alpha)}\right)^{2}\right]
\end{gathered}
$$

With this definition, the eq. (5.2)can be rewritten as

$$
Q^{\alpha}(x, t)+V+M_{x, \alpha}^{2} \frac{1}{2} \frac{\hbar^{2 \alpha}}{m^{\beta}} \frac{c^{2 \alpha}}{c^{2 \beta}} \frac{1}{\hbar^{2}}\left(\frac{\partial^{\alpha} S}{\partial x^{\alpha}}\right)^{2}-\frac{1}{8} M_{x, \alpha}^{4} \frac{\hbar^{4 \alpha}}{m^{3 \beta}} \frac{c^{4 \alpha}}{c^{6 \beta}} \frac{1}{\hbar^{4}}\left(S^{(\alpha)}\right)^{4}=-\hbar^{\beta-1} \frac{\partial^{\beta} S}{\partial t^{\beta}},
$$

deriving this equation with respect to $x^{\alpha}$, interchanging spatial and time ordering of derivatives and considering both fractional derivative orders equals, that is, $a=b$, we obtain

$-\frac{\partial^{\alpha}}{\partial x^{\alpha}}\left(Q^{\alpha}(x, t)+V\right)=\frac{\partial^{\alpha}}{\partial x^{\alpha}}\left[M_{x, \alpha}^{2} \frac{\hbar^{2 \alpha}}{2 m^{\alpha}} \frac{1}{\hbar^{2}}\left(\frac{\partial^{\alpha} S}{\partial x^{\alpha}}\right)^{2}-\frac{1}{8} M_{x, \alpha}^{4} \frac{\hbar^{4 \alpha}}{m^{3 \alpha}} \frac{1}{c^{2 \alpha}} \frac{1}{\hbar^{4}}\left(S^{(\alpha)}\right)^{4}\right]+\hbar^{\alpha-1} \frac{\partial^{\alpha}}{\partial t^{\alpha}} \frac{\partial^{\alpha} S}{\partial x^{\alpha}}$.

Defining the fractional moment as

$$
p^{\alpha}=M_{x, \alpha} \hbar^{\alpha-1} \frac{\partial^{\alpha} S}{\partial x^{\alpha}}
$$

and noting that in the lowest order in $\alpha$

$$
\frac{d^{\alpha} p^{\alpha}}{d t^{\alpha}}=\frac{\partial^{\alpha} p^{\alpha}}{\partial x^{\alpha}}\left(\frac{d x}{d t}\right)^{\alpha}+\frac{\partial^{\alpha} p^{\alpha}}{\partial t^{\alpha}}
$$

with a similar the definition of the fractional velocity [10], that relates it to a fractional linear momentum, 


$$
v^{\alpha}=\left(\frac{d x}{d t}\right)^{\alpha}=\lambda_{\alpha, \beta} p^{\alpha}
$$

with $\lambda_{\alpha, \beta}=\left(M_{x, \alpha} \frac{c^{\alpha}}{c^{\beta}}\right)^{-1}$, we shall have that

$$
-\frac{\partial^{\alpha}}{\partial x^{\alpha}}\left(Q^{\alpha}(x, t)+V\right) \equiv F^{\alpha}
$$

where $F^{\alpha}$ is defined as the fractional force. The equation above gives us a Newtonian-like fractional dynamical equation, that coincides with $\frac{d^{\alpha} p^{\alpha}}{d t^{\alpha}}$ if $\alpha=1$ and we do not consider the higher order term.

We define the fractional mechanical energy and the kinetic energy, respectively, as

$$
E^{\alpha}(x, t)=-\hbar^{\alpha-1} \frac{\partial^{\alpha} S(x, t)}{\partial t^{\alpha}}
$$

and

$$
K^{\alpha}(x, t)=M_{x, \alpha}^{2} \frac{\hbar^{2 \alpha}}{2 m^{\alpha}} \frac{1}{\hbar^{2}}\left(\frac{\partial^{\alpha} S}{\partial x^{\alpha}}\right)^{2}-\frac{1}{8} M_{x, \alpha}^{4} \frac{\hbar^{4 \alpha}}{m^{3 \alpha}} \frac{1}{c^{2 \alpha}} \frac{1}{\hbar^{4}}\left(S^{(\alpha)}\right)^{4} .
$$

In terms of these and the quantum potential, we can rewrite eq. (5.2) as

$$
E^{\alpha}(x, t)=K^{\alpha}(x, t)+Q^{\alpha}(x, t)+V .
$$

It is important to notice that, if we make $a=1$, all the results are in complete agreement with standard Bohmian mechanics with the inclusion of lower relativistic correction terms.

The expressions for the fractional moment (5.7) and the fractional energy (5.11) open up the possibility for the attainment of anomalous dispersion relations that shall be discussed as an application later on this paper.

Another point to highlight concerns energy conservation. If we assume for the phase $\mathrm{S}$ a dependence like a power of time,

$$
S(x, t)=\hbar\left(f-\omega^{\alpha} t^{\alpha}\right),
$$

where $\omega_{\alpha}$ is a multiplicative constant and $f$ is some functions depending explicitly only on $x$, then we obtain for the fractional energy

$$
E^{\alpha}(x, t)=-\hbar^{\alpha-1} \frac{\partial^{\alpha} S(x, t)}{\partial t^{\alpha}}=\hbar^{\alpha} \Gamma(\alpha+1) \omega^{\alpha},
$$

that is a constant. The the fractional energy can be conserved by an appropriate choice of phase.

\section{De Broglie relations}

In what follows, we argue that fractional De Broglie relations in the coarse-grained context naturally emerge from the energy-phase and momentum-phase relationship . 
If we write for the phase $\mathrm{S}$ a dependence like a power of time,

$$
S(x, t)=\hbar\left[k^{\alpha} x^{\alpha} \pm \omega^{\alpha} t^{\alpha}\right],
$$

we have eq. (5.15) for the energy, which is a fractional Planck-type energy relation. Its concept has already been proposed in the literature, in the context of soft matter [45]. The expression above indicates that the Planck quantum energy relation may describe a fractional package of energy, changed from the usual one by the imposed fractionality of interactions and the medium.

Notice that whenever $\alpha=1$, the usual quantum energy $E=\hbar \omega$ is recovered.

Inserting the phase $S$ into eq. (5.12) leads to

$$
\begin{aligned}
K^{\alpha}(x, t) & =M_{x, \alpha}^{2} \frac{\hbar^{2 \alpha}}{2 m^{\alpha}} \frac{1}{\hbar^{2}}\left(\Gamma(\alpha+1) k^{\alpha}\right)^{2}-\frac{1}{8} M_{x, \alpha}^{4} \frac{\hbar^{4 \alpha}}{m^{3 \alpha}} \frac{1}{c^{2 \alpha}} \frac{1}{\hbar^{4}}\left(\Gamma(\alpha+1) k^{\alpha}\right)^{4}= \\
& =M_{x, \alpha}^{2} \frac{1}{2 m^{\alpha}} \frac{1}{\hbar^{2}}\left[\Gamma(\alpha+1) \hbar^{\alpha} k^{\alpha}\right]^{2}+\mathscr{O}\left(p^{4}\right) .
\end{aligned}
$$

This suggests a definition of the momentum as below:

$$
p^{\alpha}=M_{x, \alpha} \Gamma(\alpha+1) \hbar^{\alpha} k^{\alpha}
$$

which reduces to De Broglie relations of ordinary quantum mechanics for $\alpha=1$.

\section{Anomalous Dispersion Relation for massless Particles}

Based on eq. (2.8), with $m^{\alpha}=0$, the dispersion relation for a massless particle can be written as

$$
E^{\alpha}=\sqrt{p^{2 \alpha} c^{2 \alpha}}
$$

it is a non-linear relation.

As an example, with the help of eqs. (5.15) and (5.18) one can write eq.5.19 as

$$
\left[\hbar^{\alpha} \Gamma(\alpha+1)\right]^{2} \omega^{2 \alpha}=p^{2 \alpha} c^{2 \alpha}=M_{x, \alpha}^{2} \Gamma^{2}(\alpha+1) \hbar^{2 \alpha} k^{2 \alpha} c^{2 \alpha},
$$

or simplifying, the dispersion relation can be cast in terms in terms of $\omega^{\alpha}$ and $k^{\alpha}$ as

$$
\omega^{\alpha}=M_{x, \alpha} k^{\alpha} c^{\alpha}
$$

\section{Application: Group and Phase Velocities, The Dynamical Evolution Equation for the Phase and the Medium Refractive Index}

As a general application, we develop expressions for group and phase velocities. Also, a 
simple dynamical evolution equation for the phase $S$ is obtained and a connection with refractive index of the medium is suggested.

The fractional group velocity can be written as

$$
v_{g}^{\gamma}=\frac{d^{\gamma} E^{\alpha}}{\left(d p^{\alpha}\right)^{\gamma}}=\frac{\Gamma(\alpha+1)}{\Gamma(\alpha-\gamma+1)} p^{\alpha-\gamma} c^{\alpha},
$$

and the phase velocity can be expressed as the ratio between fractional energy and momentum is

$$
v_{p h}=\frac{E^{\alpha}}{p^{\alpha}}=c^{\alpha} .
$$

Comparing both expressions, we can see that the phase velocity and the group velocity are equal only in the integer limit where $\alpha=\gamma=1$. Also, the group velocity has an non-linear behavior for non-integer fractionality. The expressions above indicate that the propagation of energetic particles in a coarse-grained medium might travel with a velocity $c^{\alpha}$, less than the speed of light $c$ in the trivial vacuum. We remark here that this was achieved without the necessity to modification of Maxwell's equations.

To the calculation of the group velocity is carried out with a different fractional order derivative parameter $\gamma$ (instead of $\alpha$ ). We argue that we may be eventually trying to describe the dynamics of a system with some slightly different fractional parameter. The fractionality of the medium may have changed slightly in some way, due to perturbation interactions. The changes, even if small, may give rise to the non-linear behavior described by the fractional expression obtained above.

With the help of the dispersion relation for a massless particle given by eq. (5.19), we can write an equation for the dynamical evolution of the phase $S$. For this, we use also equations (5.7) and (5.11). Then, we write

$$
\frac{\partial^{\alpha}}{\partial t^{\alpha}} S(x, t)+M_{x, \alpha} \frac{\partial^{\alpha} S}{\partial x^{\alpha}} c^{\alpha}=0 .
$$

The canonical momentum-energy relation may be connected with the refractive index and can be written as a reference equation, relating the photon propagation velocity in the coarse-grained medium and the standard velocity of light in the trivial vacuum. This indicates an energy-dependent vacuum refractive index,

$$
\frac{p^{\alpha}}{E^{\alpha}}=\frac{n_{\alpha}}{c}
$$

with $n_{\alpha}$ being the refractive index [46] for a coarse-grained non-trivial optical medium.

If the kinetic moments are considered, then

$$
\frac{p^{\alpha}}{E^{\alpha}}=\frac{1}{n_{\alpha} c} .
$$

The expressions above, with the help of eqs. (5.7), (5.11) and (6.3) may provide a mechanism to attain a refraction index for the medium in a coarse-grained scenario and indicate there may 
be a close connection between fractionality and anomalous dispersion relations for photons, in a coarse-grained medium. This agrees with the reported literature concerning anomalous dispersion relations for UHECR photons [52], specially with some experimental data, like the once coming from cosmic gamma ray burst measurements. It can also be compared with results from noncommutative theories [53]. We suggest that UHECR sources [54], the distant astrophysical sources such as Gamma-Ray Bursts (GRBs) and active galactic nuclei (AGNs) or pulsars [55] may be the best framework to test the model presented here, including vacuum refractive index relation.

\section{Concluding Comments}

There has been considerable interest over the past recent years in the so-called theory of "weak" quantum measurements, whose aim is to measure the average value of a quantum observable while negligibly disturbing the measured system $[56,57,58,59,60]$. Very recently, experimental observation of trajectories of a photon in a double-slit interferometer was reported, which displayed the qualitative features predicted in the De Broglie-Bohm interpretation [61, 62].

Possibilities like connections with a quantum gravity theory emerge from the fact that modified fractional Newtonian equation could be connected with a fractional Newtonian dynamics similar to MOND [63]. The natural emergence of a fractional Newtonian equation implicitly involves a nonlocal theory leading to a Newtonian law with memory, a characteristic of fractional derivatives. Also, the fractional energy reinforces the expectation of the presence of quantum effects. Those effects can be also associated with collective behavior in a fractal space-time tissue, where fluctuations can give rise to excitations like fractons in the net fractals [64].

Also, a version of fractional De Broglie relations naturally comes out from our equations and we recover the integer relations in the convenient limit. In connection with the probability conservation, in the fractional case, we have worked out, to the lowest order in the relativistic correction, the fractional probability current. The probability can be conserved in this nondifferentiable spacetime if we consider a fractional version of continuity equation that reduces to the standard one in the integer limit or, in other words, integer dimensions. As an outlook for a forthcoming work, solutions with the Mittag-Lefller instead of exponential solutions, shall be analyzed in two possibilities: nondifferentiable space of solutions and coarse-grained space-time in the argument of refereed special solution function.

We have also proposed a formulation for an anomalous dispersion relation and a refraction index, related to massless particles in a coarse-grained medium and a vacuum refractive index for a coarse-grained non-trivial optical medium, that may indicates that energetic particles in a coarsegrained medium might travel with a velocity $c^{\alpha}$ less than the corresponding one in a non-interacting vacuum with speed of light $c$. In the context of other approaches, some similar space-time "foam effect" were already reported in the scientific literature [65].

A possible connection with reported literature of non-commutative theories was suggested [53]. 
We indicate that a possible test framework for the model presented here may be UHECR sources [54], GRBs and AGNs or pulsars [55].

\section{Acknowledgments}

J. A. Helayël-Neto, A. Cherman and C.F.L. Godinho would like to express their gratitude to the Brazilian FAPERJ and CNPq for partial financial support.

J. Weberszpil and C.F.L. Godinho would like to express their gratitude to C.B.P.F. by the kindness and hospitality during our work discussions.

\section{References}

[1] Richard Herrmann, Common aspects of q-deformed Lie algebras and fractional calculus, Physica A 389 (2010) 46134622; [arXiv: physics . gen-ph/1007.1084v1].

[2] E.M.C Abreu and C.F.L. Godinho, Fractional Dirac bracket and quantization for constrained systems, Phys. Rev.E 84 (2011) 026608.

[3] Cresus F.L. Godinho, J. Weberszpil, J.A. Helayël-Neto, Extending the D'Alembert Solution to Space-Time Modied Riemann-Liouville Fractional Wave Equations, Chaos, Solitons \& Fractal, Chaos, Solitons \& Fractals, 45 (2012) 765-771.

[4] K.G. Dyall, Inclusion of Relativistic Effects in Electronic Structure Calculation, Aust. J. Phys.39 (1986) 667-78.

[5] Q. Su, B. A. Smetanko 1 , and R. Grobe, Wave Packet Motion in Relativistic Electric Fields, Laser Physics, 8, No. 1, (1998) 93-101.

[6] Markus Reiher and Bernd $\mathrm{He} \beta$, Relativistic Electronic-Structure Calculations for Atoms and Molecules, Modern Methods and Algorithms of Quantum Chemistry, Proceedings, Second Edition, J. Grotendorst (Ed.), John von Neumann Institute for Computing, Jülich NIC Series, Vol. 3, ISBN 3-00-005834-6, pp. 479-505, 2000.

[7] Nickisch L J, Durand L and Durand B,Salpeter equation in position space: numerical solution for arbitrary confining potentials, Phys. Rev D 30 (1984) 660-70;

[8] Frohlich J and Lenzmann E, Blowup for nonlinear wave equations describing boson stars, Communications on Pure and Applied Mathematics, Vol. LX, (2007) 1691-1705.

[9] G. Jumarie, J. Appl. Math. \& Computing Vol. 24, No. 1 - 2, (2007) 31 - 48; Applied Mathematics Letters 22, (2009) 378385; . Guy Jumarie, Table of some basic fractional calculus formulae derived from a modified RiemannLiouville derivative for nondifferentiable functions, Applied Mathematics Letters 22 (2009) 378385.

[10] Guy Jumarie, From Lagrangian mechanics fractal in space to space fractal Schrödinger's equation via fractional Taylor's series, Chaos, Solitons and Fractals 41 (2009) 1590-1604.

[11] Jumarie, G.; White noise calculus, stochastic calculus, coarse-graining and fractal geodesic. A unied approach via fractional calculus and Maruyamas notation, In Brownian motion: Theory, Modelling and Application, R.C. Earnshaw and E.M. Riley Edit. pp 1-69, Nova Publishing, 2011. 
[12] Jumarie, G. An approach to differential geometry of fractional order via modified Riemann-Liouville derivative, Acta Mathematica Sinica, Published online: February 13, (2012); DOI:

10.1007/s10114-012-0507-3.

[13] Guy Jumarie, Derivation and solutions of some fractional Black-Scholes equations in coarse-grained space and time. Application to Merton's optimal portfolio. Computers \& Mathematics with Applications 59(3) (2010) 1142-1164.

[14] Nottale L. Fractal space-time and micro physics. Singapore: World Scientific; 1993; Nottale L., Scale Relativity, Fractal Space-Time, and Quantum Mechanics, Chaos Solitons Fract 4 (1994) 361-388.

[15] S. C. Lim, L. P. Teo, Casimir Effect Associated with Fractional Klein-Gordon Field, arXiv:1103.1673v1 [math-ph],9 Mar 2011.

[16] M. S. Plyushchay and M. R. de Traubenber, Cubic root of Klein-Gordon equation, Phys. Lett. B 477, (2000) 276-284.

[17] A. Raspini, Simple solution of fractional Dirac equation of order 2/3, Phys. Scr.64, (2001) $20-22$.

[18] P. Zavada, Relativistic wave equations with fractional derivatives and pseudodifferential operators, J. Appl. Math. 2, (2002) 163-197.

[19] E. C. Marino, Quantum electrodynamics of particles on a plane and the Chern-Simons theory, Nucl. Phys. B 408, (1993) 551-564.

[20] G. G. Barci, C.D. Fosco and L.E. Oxman, On bosonization in 3 dimensions, Phys. Lett. B 375 (1996) 267-272.

[21] D. A. R. Dalvit and F.D. Mazzitelli, Running coupling constants, Newtonian potential, and nonlocalities in the effective action, Phys. Review D 50 (1994) 1001- 1009.

[22] R. L. P. G. do Amaral and E. C. Marino, Canonical quantization of theories containing fractional powers of the D'Alembertian operator, J. Phys. A: Math. Gen. 25 (1992) 5183.5200.

[23] D. G. Barci, L. E. Oxman and M. Rocca, Canonical quantization of nonlocal field equations, Int. J. Mod. Phys. A 11 (1996) 2111-2126.

[24] S. C. Lim and S. V. Muniandy, Stochastic quantization of nonlocal fields, Phys. Lett. A 324 (2004) 396-405.

[25] S. C. Lim, Fractional derivative quantum fields at positive temperature, Physica A 363 (2006) 269-281.

[26] S. Albeverio, H. Gottschalk and J-L Wu, Convoluted generalized white noise, Schwinger functions and their analytic continuation to Wightman functions, Rev. Math. Phys. 8 (1996) 763-817.

[27] M. Grothaus and L. Streit, Construction of relativistic quantum fields in the framework of white noise analysis, J. Math. Phys. 40 (1999) 5387-5405.

[28] Sami I. Muslih, Om P. Agrawal, Dumitru Baleanu, A Fractional Schrödinger Equation and Its Solution, Int. J. Theor. Phys. 49 (2010) 1746-1752.

[29] Nikolai Laskin, Fractional quantum mechanics and Le'vy path integrals, Physics Letters A 268 (2000) 298-305. 
[30] N. Laskin, Fractional Schrödinger equation, Phys. Rev. E 66 (2002) 056108.

[31] Xiaoyun Jiang, Haitao Qi, and Mingyu Xu, Exact solutions of fractional Schrödinger-like equation with a nonlocal term, J. Math. Phys. 52 (2011) 042105.

[32] M. Naber, Time fractional Schrödinger equation, J. Math. Phys. 45, (2004) 3339.

[33] Shaowei Wang and Mingyu Xu, Generalized fractional Schrödinger equation with space-time fractional derivatives, J. Math. Phys. 48 (2007) 043502.

[34] Kolwankar KM, Gangal AD. Local fractional calculus: a calculus for fractal space-time. In: Fractals: theory and application in engineering, Delft: Springer; 1999.

[35] Kolwankar KM, Gangal AD. Fractional differentiability of nowhere differentiable functions and dimensions, Chaos 6 (1996) 505-23.

[36] Kolwankar KM, Gangal AD., Local fractional Fokker- Planck equation, Phys Rev Lett 80 (1998) 214-217.

[37] A. Babakhani and Varsha Daftardar-Gejji, On calculus of local fractional derivatives, J. Math. Anal. Appl. 270 (2002) 66-79.

[38] Yan Chen a, Ying Yanb, Kewei Zhangc, On the local fractional derivative, J. Math. Anal. Appl. 362 (2010) 17-33.

[39] A. Carpinteri, B. Chiaia, P. Cornetti, The elastic problem for fractal media: basic theory and finite element formulation, Computers and Structures 82 (2004) 499-508.

[40] W. Chen, Time-space fabric underlying anomalous diffusion, Chaos, Solitons and Fractals 28 (2006) 923-929.

[41] W. Chen, Hongguang Sun, Xiaodi Zhang, Dean Koroak, Anomalous diffusion modeling by fractal and fractional derivatives, Computers and Mathematics with Applications 59 (2010) 17541758.

[42] Kobelev V., On a Leibnitz-type fractional derivative, arXiv: math-ph/1202.2714; The variant of post-Newtonian mechanics with generalized fractional derivatives, Chaos 16 (2006) 043117.

[43] Ralf Metzler, Generalized Chapman-Kolmogorov equation: A unifying approach to the description of anomalous transport in external fields, Phys. Rev. E 62 (2000) 5.

[44] Adrián A. Budini and Manuel O. Cáceres, Functional characterization of generalized Langevin equations, J. Phys. A: Math. Gen. 37 (2004) 5959-5981.

[45] W. Chen, An Intuitive Study of Fractional Derivative Modeling and Fractional Quantum in Soft Matter, Journal of Vibration and Control 14: 1651 (2008).

[46] Stephen M. Barnett, Resolution of the Abraham-Minkowski Dilemma, PRL 104, 070401 (2010).

[47] Marco Buiatti, Paolo Grigolini and Anna Montagnini, Dynamic Approach to the Thermodynamics of Superdiffusion, Phys. Rev. Lett. 82 (1999)17 3383-3387.

[48] Gerardo Aquino, Mauro Bologna, Paolo Grigolini and Bruce J. West, Aging and rejuvenation with fractional derivatives, Phys. Rev E 70 (2004) 036105.

[49] C. Tsallis, S. V. F. Levy, A. M. C. Souza, and R. Maynard, Statistical-Mechanical Foundation of the Ubiquity of Levy Distributions in Nature, Phys. Rev. Lett. 75 (1995) 3589-3593. 
[50] Robert Caroll, On fractional quantum potential, Progress in Physics 2 (2012) 82-86.

[51] K.Miller and B. Ross, An Introduction to the Fractional Calculus and Fractional Differential Equations, Willey\&Sons, INC., 1993.

[52] Roberto Aloisio, Pasquale Blasi, Piera L. Ghia, and Aurelio F. Grillo, Probing the structure of space-time with cosmic rays, Phys. Rev. D 62, (2000) 053010 .

[53] Wolfgang Bietenholz, Could the photon dispersion relation be non-linear?, Fortschr. Phys. 57, No. 5 $-7,(2009) 505-513$.

[54] Cong-Xin Qiu and Zi-Gao Dai, Testing Lorentz violation using propagating UHECRs, Research in Astron. Astrophys, 9, (2009) 4, 409-421.

[55] John Ellis, N.E. Mavromatos, D.V. Nanopoulos, Probing a possible vacuum refractive index with $\gamma$-raytelescopes, PhysicsLettersB674(2009)83־86.

[56] Yakir Aharonov, David Z. Albert, and Lev Vaidman, How the result of a measurement of a component of the spin of a spin-1/2 particle can turn out to be 100, Phys. Rev. Lett. 60 (1988) 1351-1354.

[57] Y. Aharonov and L. Vaidman, In: Time in Quantum Mechanics, J.G. Muga et al. eds., (Springer) 369-412 (2002); [quant-ph/0105101] .

[58] O. Hosten and P. Kwiat, Observation of the spin Hall effect of light via weak measurements, Science 319 (2008) 787.

[59] J. S. Lundeen, A. M. Steinberg, Experimental Joint Weak Measurement on a Photon Pair as a Probe of Hardy's Paradox, Phys. Rev. Lett. 102 (2009) 020404.

[60] K. Yokota, T. Yamamoto, M. Koashi, N. Imoto, Direct observation of Hardy's paradox by joint weak measurement with an entangled photon pair, New J. Phys. 11 (2009) 033011.

[61] Sacha Kocsis, Sylvain Ravets, Boris Braverman, Krister Shalm, Aephraim M. Steinberg, Observing the trajectories of a single photon using weak measurement, 19th Australian Instuturte of Physics (AIP) Congress, 2010.

[62] Sacha Kocsis, et al., Observing the Average Trajectories of Single Photons in a Two-Slit Interferometer, Science 332, 6034 (2011) 1170-1173.

[63] Mordehai Milgrom, MOND-a pedagogical review, Acta Phys.Polon. B32 (2001) 3613; [ [arXiv:astro-ph/0112069v1].

[64] Z. Bak, Fracton oscillations in the net fractals Materials Science-Poland, . 25, No. 2 (2007) 491-496.

[65] John Ellis, N.E. Mavromatos, D.V. Nanopoulos Probing Models of Quantum Space-Time Foam, arXiv:gr-qc/9909085v1. 